\title{
MULTIPLE GANGRENE OF THE SKIN IN INFANTS
}

\author{
AND ITS CAUSES.
}

\author{
$\mathbf{B Y}$ \\ H. RADCLIFFE CROCKER, M.D., F.R.C.P., \\ PHYSICIAN TO THF SKIN DRPARTMENT OF UNIVERSITY COLLEGR HOSPITAL; \\ PHYGICLAN TO THE BAST LONDON HOSPITAL FOR OHILDREN.
}

Received March 8th-Read June 14th, 1887.

THE occurrence of multiple gangrenous lesions of the skin in children has been noted from time to time by various authors, but our knowledge of the significance of most of these cases is chiefly due to Mr. Jonathan Hutchinson, who showed that gangrenous ulceration was a rare complication of varicella, and, subsequently, that it might still more rarely, also follow vaccination.

I hope to carry the matter further by the cases I am about to relate, and demonstrate that even this is not the whole truth, but that other lesions, not in any way of a specific character, may also give rise to this serious condition.

Secondly, that there are various grades of the process after varicella, some of them so mild as to be only slightly ulcerative. In these, the eruption comes out in crops and may keep up the disease for a considerable time. These cases are generally attended with severe 
itching, and appear to me to correspond with the cases called by Mr. Hutchinson "varicella prurigo."

Thirdly, a precisely analogous condition may follow vaccinia.

Fourthly, I would suggest that the condition is of microbic origin and may follow any pustular eruption in which the soil is suitable. The predisposing conditions are tuberculosis, as was first pointed out by Dr. Barlow, while congenital syphilis and rickets are probably also etiological factors in a certain number.

\section{Class 1.-No Antecedent Varicella.}

CASE 1.-Ellen A. B-, æt. 1, was first brought to University College Hospital as an out-patient on July 7th, 1886.

The family history was good except that one maternal aunt died of phthisis, ascribed to hard work at the washtub, but the child was suffering from well-marked rickets, had some bronchitis and profuse sweating. Three weeks previously, an eruption had commenced on the neck and spread all over the body, and when seen, every part except the face, hands, and feet was more or less covered, most thickly on the chest, scapula and forearms, with red papules, from a pin's head to a hemp-seed in size, most of them flat, angular, and shining, but some, chiefly on the thighs near the knees, were round and convex. On the largest and deepest coloured of these last was a minute pustular centre. There was very little, if any, itching. The child was shown to the Dermatological Society on July 14th as a case of lichen planus infantum with miliaria rubra on the thighs, and the diagnosis was not disputed.

It was in these miliaria lesions near the knees, that the more serious lesions commenced on July 16 th and 17 th. At the same time some more mattery heads appeared upon the buttocks, which broke and became sores by the 18 th. She was brought again to the hospital on the 21 st. There 
were then many small ulcers on the buttock and lower part of the abdomen, two on the forearm, one about one third of an inch in diameter in front of the thigh (the first to be formed), and two above the knee on the left side.

The child was taken into the hospital on July 24th : she was fairly well nourished but had a good deal of bronchitis, nearly all over her chest.

Distribution.-The lichen papules on the upper part of the body had not entirely gone. There was nothing on the front of the scalp, but on the left side, on a level with the ear, were some small superficial excoriations covered with crusts, and a few also on the right side. There was also an irregular group of ulcers, from one eighth to three quarters of an inch in diameter, scattered irregularly over the back of the head in the left mastoid region and immediate neighbourhood, and a few also on the occipital region.

On the right side of the face were about half a dozen superficial ulcers about one eighth of an inch across. On the right arm were six or eight crusted excoriations, scattered irregularly over both surfaces. All but two were quite superficial sores; of these, one was a pustule one sixteenth of an inch across, with red areola, and the other a lesion with a dark central crust one eighth of an inch across, slightly depressed below the surface, also with a red areola. On the left arm were two deep ulcers, two thirds of an inch in diameter, on the flexor aspect, and two about a quarter of an inch in diameter. On the wrist there were two smaller ones, one on the upper arm, three on the outer surface, and two superficial on the elbow tip; the arms were free.

There were a few superficial sores on the chest and on the right clavicle, and on the lower ribs on the left side, was an irregular group of five, not very deep, and from an eighth to a quarter of an inch in diameter. All these were covered with a reddish-brown scab, sunk below the surface, and one of them had a pustular margin and 
resembled a vaccine vesicle. Immediately below the ribs, was a flat pustule a quarter of an inch across, with a narrow red areola. On the abdomen only three or four lesions were present above the umbilicus, but there were a large number scattered below it, most of them scabbed, and some with pustular margins, but the scabs were simply dried secretion. There were also a few pustules from a pin's head to a hemp-seed in size, some flat, some convex, and on the right flank was an excavated ulcer three quarters of an inch across. On the anterior surface of the thighs were a few small lesions on the upper part, but on the left near the knee were two sharp-cut ulcers half an inch across and a quarter of an inch deep in the centre. On the right thigh, rather higher up, was a somewhat larger and deeper ulcer and a smaller one near it. On the outer side of the thighs the ulcers were very deep and numerous, thickly aggregated in the upper two thirds, and varying from an eighth to three quarters of an inch in diameter.

They were less numerous on the left than on the right side ; the left leg below the knee was free, but the right, had two on the front of the knee and about half a dozen lower down. On the back were a few comparatively superficial and small lesions at the neck, and over the scapulø, and three or four in other parts scattered irregularly. There were two large deep ulcers on the loins, but the greater number were on the buttocks and upper two thirds of the thighs, especially the right. On the buttocks they were so thickly placed that they coalesced in one place on the left buttock into an ulcer an inch and a half long, and half an inch wide, the edges were sharply cut, sinuous and undermined, and the base deep and irregular. These ulcers, having been kept moist with the napkin, were not covered with scabs, but none of these were granulating, and there were more on the left than the right buttock, but the deepest were on the right side. The anus and its immediate neighbourhood were quite free. With the exception of two ulcers just below the left calf, the back 
of the left leg was free from the upper third of the thigh downwards, while they were sparsely scattered all over the right leg and thigh. The palms and soles were free.

Observation showed that the lesions were produced in the following way :-A pustule about a sixteenth of an inch formed, with red areola, enlarged to an eighth of an inch, then dried into a scab, surrounded by a ring of pus; ulceration proceeded laterally and vertically below the scab to a varying extent, and a slough was formed, the centre becoming quite black. When this slough was thrown off, it left a sharply-cut roundish or oval ulcer with slightly irregular margins, sometimes undermined, sometimes going abruptly down to the deepest part of the ulcer, at others sloping obliquely down towards the centre. The ulcers never spread after the separation of the slough, and they were of all depths and sizes, from a superficial excoriation to a very deep ulcer. The largest single ulcer was about three quarters of an inch in diameter, and about a third of an inch at its deepest part, but there were some compound ones larger than this.

Wet boracic lint was ordered for the buttocks, but as the patient did not seem comfortable with this, iodoform wool was applied, and iodo-vaseline for the rest of the body. The wool, however, adhered too closely, and gave so much pain on removal that iodoform powder at first, and afterwards iodol sprinkled on were substituted. A few fresh pustules appeared at intervals and ran the course already described. On August 9th there was diarrhœa; a few fresh lesions had formed, but most of the sloughs had separated, and on the 11th it was noted that while the ulcers on the buttocks were deeper, there were no fresh lesions. The diarrhœa continued until death on August 19th, there having been no fresh lesions since the 11th. The temperature chart ranged from $99^{\circ}$ to $104^{\circ}$. I was away for the vacation during the last ten days of life, and the post-mortem was made fifteen hours after death by Mr. Campbell Williams in Dr. Barlow's presence. Post-mortem.-Body much emaciated, rigor mortis prevOL. LXX. 
sent. On the thorax there were some signs of repair in the ulcers, but those on the buttocks were very deep, clean cut, and undermined. On the scalp they were not numerous, but two of them, the size of a florin and
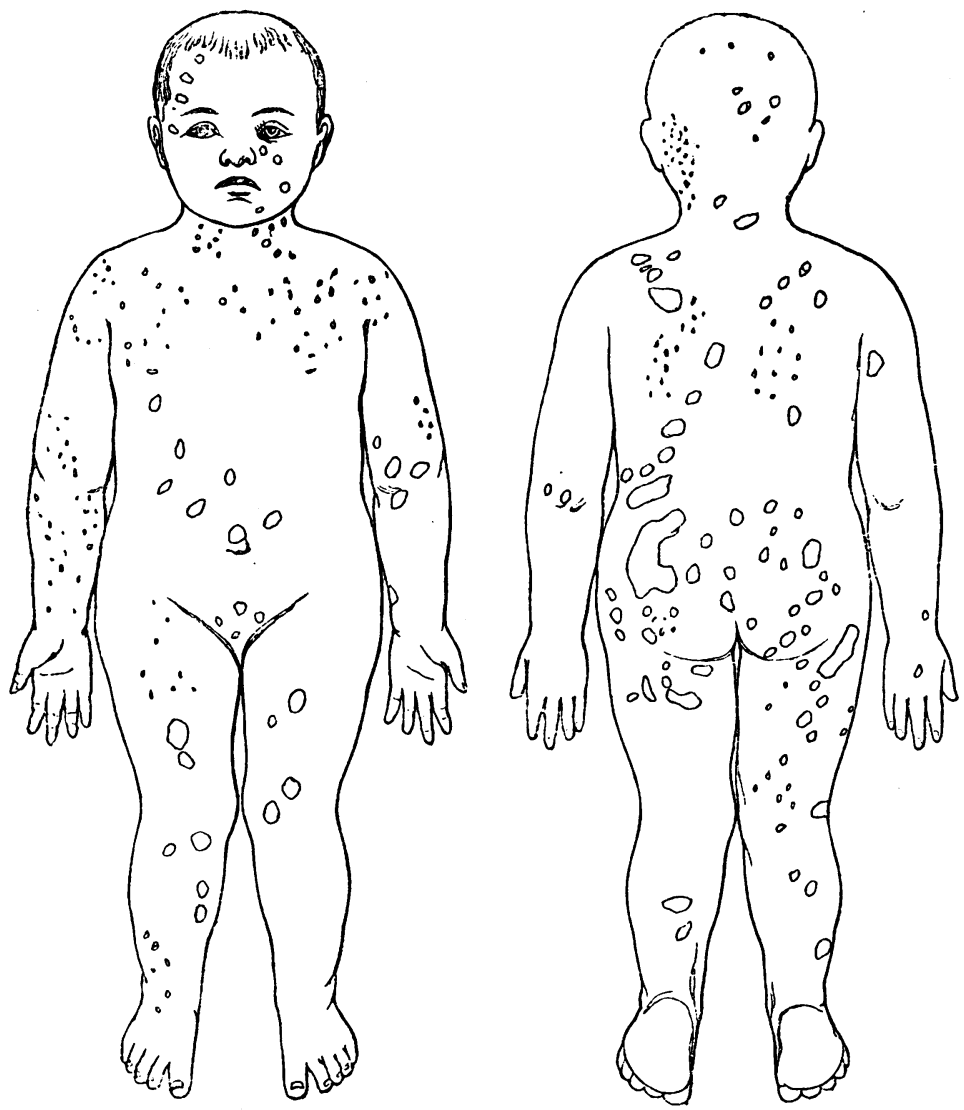

half a crown respectively, in the occipital and left parietooccipital regions, went down to the pericranium. There was no attempt at symmetrical distribution anywhere except on the thorax. 
On opening the body, the pleurm were healthy, the lungs were consolidated at both bases, especially on the right side, and much congested, and there were one or two tubercles in both lungs. The bronchial glands were about the size of an almond and caseous. The liver was enlarged and fatty, the mesenteric glands were large and infiltrated with tubercular deposit; all the other viscera were normal.

As the above account is somewhat meagre, Dr. Barlow has been kind enough to supplement it from a few brief notes of his own.

"Some of the lesions on the scalp had ulcerated so deeply that there was a thin horny floor which was firmly adherent to the subjacent bone, but the bone was not actually bare."

"In the thorax, the bronchial glands were caseous and enlarged, the lungs were emphysematous in front, and the lower lobes at the back showed extensive areas of bronchopneumonia which had become partially caseous. On the under surface of the upper lobes were some groups of undoubted miliary tubercle. There was a small vomica almost certainly due in part to softening of one of the bronchial glands which penetrated into the root of the lung at the lower lobe on one side, and close to it was another caseous gland. The mesenteric glands were enlarged and caseous, and in the intestines some of the Peyer's patches showed small deposits of tubercle with very early commencing ulceration."

I have four other cases in which there is no reason to believe that varicella had any connection with the ulceration. In one of these (Case 3) the eruption was bullous and may be considered to belong to a different category, but at all events there was deep ulceration and no sign of varicella, and $I$ therefore record it here. In Case 4 the evidence against a varicella connection is very strong, I should say conclusive, and in Case 5, a severe and fatal one, not only did it begin on the lower extremities, but the mother averred that she was acquainted with chicken- 
pox eruption, and that the antecedent lesions did not at all resemble it.

CASE 2.-Mary A. C-, æt. 6 months, was admitted as an out-patient at the Shadwell Hospital on February 27th, 1882. She was vaccinated when two weeks old; there was no evidence of varicella, there were no physical signs in the chest; she had been suckled but was much emaciated. The eruption began in the small of the back, two weeks before she came to the hospital ; it was limited to the trunk, except one small ulcer outside of the left leg below the knee. It was abundant on the buttocks and reached half way up the back, where it was to some extent symmetrical. In the front of the body, on the right side only, there were five ulcers and a few scars; there were one or two on the head, and many scars from previous lesions in various parts. Those in front were scabbed superficial ulcers half an inch in diameter with a bright red areola; there were a few pustules like small boils which began as minute pustules on an indurated inflamed base, the whole being the size of a No. 3 shot.

The treatment consisted of sulphide of calcium internally, and diluted ammonio-chloride of mercury ointment applied to the sores. On March 27th all the ulcers were healed and the child was thriving, but an abscess having formed on the back she was admitted into the hospital, where she died on May 7th, but I have been unable to obtain particulars of the post-mortem.

CASE 3.-Adelaide $\mathrm{S}$-, æt. 3 years, was admitted as an out-patient at the Children's Hospital June 25th, 1883. The disease began nine days previously with a bulla half an inch across, then others formed and began to ulcerate. When first seen, there were bullæ half an inch across, containing clear serum and without any areola; there were pustules from an eighth to a quarter of an inch across with an areola; superficially ulcerated patches covered with a layer of pus; deep ulcerations, one very deep, on the site of the first bulla, three quarters 
by a third of an inch in area, nearly all of the lesions being confined to the right side of the thigh and leg. During the previous two days several minute pustules had formed, a few on the inner side of the left thigh and on the right side of the head. The child was slightly rickety, fairly well nourished, but she had been pulled down by a continual diarrhoea which had only been stopped for a fortnight. The mother had lost four children out of eleven, three of whom died of " consumptive bowels;" "they seemed to drop off at three months and waste, but they none of them had any rash." The case was diagnosed at the time as an "ulcerating pemphigus."

Case 4.-Louisa $\mathrm{M}$-, æt. 3 years, was admitted as an out-patient to the Shadwell Children's Hospital on May 12th, 1884. The child had had "glass-pock" eight months previously and whooping-cough had been present for a month. One week before she came to the hospital a white patch formed on the occiput with blood in the middle following two pea-sized vesicles full of water; others followed on the scalp and on the right second finger. On the right parietal region when first seen was a white patch about a square inch in area slightly depressed below the surface, looking as if the skin was undermined with the border slightly raised and vesicular. Near it, was a millet-sized vesicle with a faint red line round it and there was also an areola round the large one. Near the white patch in the middle of a half inch vesicle was a scab, and there was also on the occiput another scabbed patch with blood-stained crust, about an inch and a half in diameter ; there were several smaller lesions with black sloughy centres about the scalp, and a deeply excavated ulcer an inch in diameter over the right ear, and the lips were scabbed and had superficial ulcers upon them.

CASE 5.-Ellen A. H-, æt. 16 months, came first to University College Hospital on February 17th, 1880. The father was consumptive, the mother well, but lived poorly. She had had nine children, eight of whom were living, 
and three miscarriages, but not consecutive ; the eldest, a boy, æt. 17, had had some cervical abscesses, but the others were healthy. The child before the patient, died when twenty months old, and had similar lesions on the skin for four months before death; it had improved under my colleague Mr. Barker's treatment, although it subsequently died of bronchitis. The spots first came on the right leg, and then spread upwards. The following notes were taken on March 17th :-The patient was fairly nourished, but suffered from enlarged cervical glands. She was vaccinated when six months old, and the eighth or ninth day afterwards, small red pimples came out over the whole body, which "felt like a nutmeg-grater," but this did not last longer than a month. A fortnight before she came to the hospital a few spots came on the right leg, preceded by fretfulness, then others came in a corresponding position on the left leg, fresh ones continued to come out, extending from below upwards to the buttocks, and then over the body, ulcerating soon after their formation. When first seen the bulk of the eruption was papular, while pustules existed round the hair-follicles of the legs. There was an eczematous patch about an inch in diameter on the head; there had been some eczema behind the ears, and otorrhœa.

The distribution of the lesions was as follows :-There were the remains of a good many on the scalp, but they had not been of a severe kind, and they were dry and nearly well when the child was seen; there were about a couple of dozen on the back of the forearms, and a few on the front, and on the upper arm, but the palms and soles were free. The upper two thirds of the trunk were almost free, but there was some desquamation on the lower third. They were comparatively numerous, increasing in number and size from above downwards, being pretty freely distributed over the buttocks and thighs, and densely crowded from below the knees to the feet, the lesions as a whole being roughly symmetrical. The course of the eruption is as follows:-It begins as slightly red, pin's-head sized, flattish 
papule, with only an indication of suppuration at the apex. As it enlarges, the pustalation becomes more distinct, but the lesion is still flat at the top. When it is about an eighth of an inch in diameter the pus dries into a scab; most of the lesions gradually get well; others, however, especially on the feet and legs, have a pustular border formed round the scab, and this again has an areola, the whole somewhat resembling a vaccinia lesion. This soon ulcerates beneath the scab, forming a sharply punchedout flat-bottomed ulcer, with a ring of inflammation round. Others again are much deeper, a black slough having formed which, after separation, leaves a clean-surfaced but deep ulcer; or the smaller ulcers may extend peripherally and vertically, and then are irregular at the base, though the outline is generally roundish or oval, unless neighbouring lesions have coalesced. There were no ulcerations on the head and face, a few superficial ulcers on the forearms, and one or two on the trunk. On the thigh and buttocks were several of large size, but the ulcerative process was most marked below the knees, those on the feet being the deepest and largest, while the lower third of the leg was fairly riddled with ulcers, varying from a quarter to more than an inch in diameter, and some of them nearly a third of an inch deep. The child was not seen again, having died a few days later in convulsions. No post-mortem could be obtained.

I have next a series of six cases of varying grades of severity all accompanying or following undoubted varicella. The first one was of the most virulent kind.

\section{Class 2.-Antecedent Varicella.}

CASE 1.-Georgina S-, øt. 7 months. Both father and mother were syphilitic. The child was always weakly, and snuffled, but presented no other evidence of syphilis. On October 31st she was unwell ; on November 1st a vesicular rash appeared on the head and back which was evi- 
dently varicella; in a few days it dried into scabs. On November 3rd a black blister formed on the inner side of the right knee, which afterwards became a large gangrenous ulcer; the slough was one inch by three quarters in size. On the evening of November 3rd, and the morning of the 4 th, the mother noticed that all the vesicles were becoming black blisters. She was admitted into University College Hospital on November 8th. The child was small, feeble looking, and very drowsy. On the right knee, as already described, was a deep ulcer, another on the flexor aspect of the left knee, several on the buttocks, and a small one on the left ankle. Just above the pubes was a patch consisting of a central scab surrounded by a vesicle, and this by a red areola. It began on November 5th. On the left side was a patch formed on November 6 th ; in this only a faint areola was present, the vesicular part was more distinct, and the scab was smaller and lighter coloured.

On the buttock several small red points showed signs of vesiculation. On November 14th the temperature was $99.8^{\circ}$, there were no new sores, and the old ones had not spread. On the 15 th the temperature reached $105.2^{\circ}$ at 7 a.m. ; it fell to $97 \cdot 6^{\circ}$ at 7 p.m., and the child was weak and listless. On the 16 th the temperature rose again to $104^{\circ}$ at 2 a.m., and the child died at 7 a.m.

Post-mortem.-The upper lobe of the right lung was filled with minute infarcts the size of radish seeds, most of them breaking down in the middle. In the left lung there was broncho-pneumonia at the base. The liver was fatty. The skull-cap was thick, rough, and soft at the anterior part of the vertex, evidently due to syphilis. The brain was normal. There was no evidence of tubercle anywhere. A closely analogous case to this is recorded by Mr. Warrington Haward in the 'British Medical Journal' for 1883, in which the ohild died with pyømic lesions in the lungs.

In the next case the ulcers appeared when the varicella 
was nearly well, and only two of them were deep and gangrenous.

CASE 2.-Ruth B-, æt. 3 years. No history of phthisis in the family. The varicella broke out three weeks before I saw her, and when the pustules were nearly well sores appeared on the face and on the back of the ears.

On the abdomen and lower rib margin and over the left rectus were two dark, sloughy spots with a red areola, one of them rather deep; there were several red marks on the head and face from the previous eruption.

In the third and fourth cases all the lesions were superficial and developed on the pock pustule.

CASE 3.-William N-, æt. 7 months, came as an outpatient to Shadwell on October 31st, 1881. There was no phthisis in the family, nor any evidence of it in the patient. The eruption had been out for three weeks. It began as little spots all over like "glass-pock" on the head and back, and the sores began a week before admission.

The pocks never got well, but sores came where the scabs had been commencing on the head. When first seen, the lesions were in the following positions :-On the temples, there was an ulcer on each side, the largest about a third of an inch across, and of conical shape. There were a few varicella scars on the cheeks, and on the scalp were many pustules the size of those of varicella, and a few ulcers a third of an inch across. There were two or three scars on the forehead, and one pustule. On the left arm was an ulcer half an inch across and a quar. ter of an inch deep, the slough having separated, and there was a corresponding lesion on the right arm. The back was covered with varicella scars, and there were two or three papules with a tendency to pus formation on the top ; there were also some fresh papules on the right cheek and leg. The eruption was attended with considerable itching. Quinine internally and iodoform externally wtre 
prescribed, and when seen again on November 4th the old lesions were healing, but some fresh ones had appeared on the forehead, face, and arm, with a few on the trunk and legs, the number being greatest on the extensor surfaces.

The new eruption was in pale red papules a pin's head in size and upwards, some vesicular and dried into scabs, a few a quarter to half an inch across and beginning to ulcerate. The legs and arms were rather thickly covered with small papules; the larger ones came out at once and did not form from small ones; they all itched violently. The elder brother, who had had varicella when he was seven months old, was always kissing the patient, and several papules had appeared on his cheeks and chin, which increased in size the following week, but did not ulcerate. The patient went on very well until November 8th, when catarrhal pneumonia supervened, probably from exposure in his journey to the hospital, and he died on December 2nd. There was no autopsy.

I have met with two cases in connection with vaccination corresponding with the milder cases of ulcerating varicella, although in one place the ulcer was large and deep, which would remove doubts, if any existed, as to the similar character of the eruption.

Class 3.-Ulcerating Eruptions after Vaccination.

CASE 1.-Henry B. H-, æt. 10 months, was brought to the Shadwell Children's Hospital on January 23rd, 1882. The mother was dying of phthisis. The patient was thin and delicate. Three weeks after vaccination, the ears and one nostril began to discharge, and the right eyelid became inflamed. The eruption began on the head a week later as red pimples, the size of a small pea or less, then enlarged, and pustules formed on the top one quarter to three quarters of an inch across; then the head became 
covered with scabs a quarter of an inch across. When first seen, the head was covered with scabs from a quarter to one inch across, but with little or no inflammation round, and numerous scars of previous eruption were present. There was one inflamed pustule on the left forefinger, and a deep ulcer three quarters of an inch by half an inch over the left ischial tuberosity. There were a few on the back and legs, one just coming, consisting of a flat pustule one eighth of an inch across, raised on a red base, and the toes had been affected. There were many scars, but no attempt at symmetry. On January 30th the child was brought again, and nearly all the ulcers, except the large one over the hip, had healed.

I have some very imperfect notes of the other case, in which a red pimply eruption came out one week after vaccination, and lasted a week; it was followed by a vesicular rash, which came first on the shoulders, and then down the arms and legs, feet, palms, and 'soles. There were also some on the back of the head, and a few down the back. The vesicles became pustules with a red areola, were from a millet seed to a pea in size, and accompanied by intense itching.

There are thus a dozen cases of varying degrees of severity, some so mild and chronic, that were it not for intermediate links we should hesitate to class them with those gangrenous cases at the other end of the chain, which are fatal in a few days.

From a consideration of these and twenty-one other cases published by Mr. Hutchinson and other authors, we may draw up the following general account of the disease.

The place of onset, and mode of development varies according to whether it appears early or late in the course of the varicella, or is independent of that disease.

If it begins while the varicella lesions are still present, it commences on the head or upper part of the body, and instead of the scab being thrown off from the pock, ulce- 
ration takes place beneath it, and often a pustular border with a red areola is formed, the whole resembling a vaccination pustule, the process extending both in depth and peripherally; a black slough is formed from a quarter to an inch or more in diameter, the smaller ones, still with a pustular border and areola: after attaining a certain size, varying much, the process of separation sets in, and when completed, a sharp-edged, roundish or oval conical ulcer is formed deep or shallow, in proportion to the diameter of the slough, some of the largest being quite three quarters of an inch in the centre. Extension of the ulcer seldom takes place after the separation of the slough has commenced. When the lesions are closely aggregated coalescence will probably ensue and then very large ulcers, irregular both in contour and floor, are produced. If any fresh crops are formed, or when it commences after most, if not all, of the varicella lesions have cleared off, perhaps a fortnight or more from the onset, or in cases following vaccination, or otherwise unconnected with varicella, the ulcerative lesions usually commence on the lower half of the body. They especially affect the buttocks and thighs, as a pin's-head sized papulo-pustule, which extends to the size of a pea or larger, ruptures, and, except on the buttocks or wherever it is kept moist, dries in the centre to a scab, with the pustular border and red areola like vaccinia, and from this point follows the same course as those which started in a varicella pustule. In some cases the buttocks and parts in contact with the napkin, and sometimes the legs and the thighs, are fairly riddled with ulcers of all sizes, shapes, and depths. On the trunk and rest of the body they are not usually numerous, and though some may be very large and deep the majority are comparatively superficial.

Where the lesions are numerous and deep there is naturally much constitutional disturbance, the temperature ranging up to $104^{\circ}$ or even higher. Lung complications, tubercular, pyæmic, or inflammatory, are very frequent, and determine or hurry on the fatal issue. Should the 
child survive it is surprising how rapidly the lesions cicatrize, of course leaving deep and indelible scars.

There are all grades of the disease. In the mildest form ulceration may be quite superficial, the lesions reaching to the vaccinia-like stage and then drying up, often accompanied by pruritus, and lasting by successive crops for a considerable period; there may also be simultaneously observed mere excoriation up to pretty deep ulceration, with or without a few lesions going on to gangrenous sloughs; while in the most extreme cases hæmorrhage occurs into all the vesicles, which become rapidly gangrenous and lead to the death of the child in a few days, either with a general tuberculosis, or, pyæmic lesions. When less severe than this the contents of the vesicles are not hæmorrhagic, but a large proportion of them become gangrenous, and death is very likely to ensue but may be deferred for some time, and is usually due to a secondary complication.

As regards etiology, all the cases hitherto recorded have occurred in infants or young children; an analysis of my own and eleven of other authors, in which the age is stated, shows that by far the majority occur under one year, the figures being fourteen not exceeding one year, six not exceeding two years, and three under three years of age ; the youngest was three months old.

My colleague Mr. R. W. Parker, had a case of a girl, æt. 12 , in whom a hydroa was aggravated by the administration of iodide of potassium, into hæmorrhagic bullæ which then discharged and gave rise to extensive ulceration and sloughing lesions very suggestive of the disease under consideration. By far the majority occur in girls : fifteen out of the twenty-one cases where the sex is mentioned, and of my own cases ten out of twelve were females. With regard to the diseases antecedent to it my own cases are alone available for reference, most of the other reporters of cases having accepted Mr. Hutchinson's dictum that they were all consequent on varicella or vaccinia. In one after vaccination, a mild case, and in 
five others there was not the slightest evidence of varicella, and in one the child had been under close observation for lichen planus infantum, and the ulcerative lesions appeared to develop on miliaria rubra pustules. These facts suggest that under certain circumstances any eruption of isolated pustules may be the starting-point of the ulcers. Among predisposing causes tuberculosis has been present in so many that, as Dr. Barlow first pointed out, it must be more than a mere coincidence. In one of my fatal cases congenital syphilis was present, in another rickets, while a few were apparently quite healthy. Gangrenous ulcers of probably similar character occur sometimes as a complication of variola in adults, as well as in children.

The diagnosis is not difficult, with or without a history of varicella ; the occurrence of numerous gangrenous alcers in a young child, or even of deep ulceration beginning as a pustule, enlarging, drying into a scab in the centre, and then ulcerating, form a group of symptoms quite unmistakable.

The prognosis is serious in proportion to the tender age of the infant, the number, depth and extent of the lesions, the amount of constitutional disturbance, the presence of tuberculosis, pyæmic, or inflammatory lung symptoms.

The treatment must be general and local; quinine in one- or two-grain doses in milk every four hours is often serviceable. In some of my cases sulpho-carbolate of soda in five-grain doses every three hours has been apparently beneficial. All complications must be treated as they arise.

Wet boracic lint under oiled-silk until the sloughs have separated, and subsequently iodoform or iodol vaseline will keep the ulcers antiseptic. Freshly-made iodide of starch paste, painted on, is another convenient application, and Pasteur, of London, found a warm solution of chlorinated lime on lint give most relief. These measures and the administration of concentrated, or, in young infants, 
partially digested foods, and putting the patient in the best hygienic conditions, offer most chance of success.

I desire now (by way of appendix) to call attention to certain local gangrenes of the skin in children, especially of the genitalia and neighbourhood, of which the attending phenomena present some analogies to the cases hitherto considered.

\section{Crass 4.-Local Gangrenes of the Skin.}

Cass 1.-Elizabeth $\mathrm{H}$ - æt. $2 \frac{1}{2}$ years, out-patient at Shadwell, June 23rd, 1882. She was quite well up to the 17th, when "gatherings as if she was chapped" appeared on the ears, and then the vulva was attacked. When first seen there was superficial ulceration behind and above both ears, and on the upper part of the auricle itself, the right side being worse than the left. There were some isolated pustules over the inferior maxilla, one of which was ulcerating. The whole vulva was in a state of extreme inflammation, swollen, dusky red, especially at the lower part, where there was superficial ulceration, and also between the labia; there was also a scanty, thick, purulent discharge from the vulva. The child was fairly nourished but looked ill, and her temperature was $101^{\circ}$. She was admitted into the hospital and recovered without any further ulceration.

It may be objected to this case that it is not in the same category as those in which there is sloughing, but I think she only just escaped this by timely treatment.

Casz 2.-Florence B-, æt. 1 year and 6 months, outpatient at Shadwell, admitted October 19th, 1883. She had measles three months previously; she got over it all right, but subsequently her chest was affected, and she was treated for "bronchitis and inflammation," and when I saw her she had sonorous and sibilant râles all over the 
chest, but no dulness. Two weeks before admission pimples like little blisters appeared round the anus, then spread forwards to the top of the privates; these broke, ulcerated, and fresh ones appeared daily for four days until they were so crowded that they coalesced. On admission there was a deep ulcer two inches by three quarters on the left buttock, and at the side of the anus. Further forward, and separated by a narrow interval of healthy skin, was another irregularly bordered ulcer, which reached up to an immensely swollen vulva. The lower two-thirds of the left labium was in an ulcerating and sloughing condition, and on the right buttock was another smaller ulcer the size of a shilling, and there were two more on the right labium; the parts round were of a deep dusky red and much swollen. The child looked very ill and she was taken into the hospital under Mr. Reeves. He made incisions into the tissues where there was most tension, and applied iodoform dressings, but the child died of asphyxia on October 26th, and no post-mortem could be obtained.

CASE 3.-George R-, æt. 2 years, out-patient at the East London Hospital for Children, admitted December 15th, 1884. He had had measles five weeks previously, and got on well up to two weeks ago, when little white spots like blisters, but containing " mattery-looking stuff," with the skin round them reddened, came in the groin. They burst and became open sores, were poulticed, and the ulcers spread rapidly; the flesh looked black, and had become much worse the last two days. When seen there was, in the left inguinal region, at the bottom of the abdomen, a deeply excavated and undermined ulcer about three quarters by half an inch. In the left groin there was a deep slonghing ulcer three inches long by three quarters of an inch wide, and quite half an inch deep in some parts ; it extended on to the scrotum, but was more superficial there; on the inner side of the leg it had gone down to the subcutaneous tissue. Half an inch below the large one was an oval ulcer half an inch long, very deep, and 
with undermined edges which represented an earlier stage of the process, the large one having been formed by the coalescence of four or five "blisters" the size of a pea, and without any special arrangement with regard to each other. At the root of the penis in front was a superficial excoriation produced by scratching, but the child seemed to be in no pain. The temperature was $100.4^{\circ}$ in the rectum. There were some subcrepitant râles at the bases, but no dulness. The mother said he had quite recovered after the measles, ate well, and ran about until these ulcers appeared. His subsequent history is unknown.

CASE 4.-Emma L-, æt. 14 months, admitted on November 29th, 1886, as out-patient at Shadwell. Ten days previously two little white spots the size of a pea appeared in the groin; they were at first full of water but soon burst, and the mother applied fuller's earth and poulticed them. On admission there was on the thigh an ulcer the size of a hemp seed, and a red areola round it; it had begun the day before as a red spot, and was like the others were at first. On the lower border of the groin, extending down to the vulva, was a chain of ulcers with yellowish sloughs and inflamed base; the largest was about one inch long and a quarter of an inch wide, and the next largest three quarters by half an inch, and lower down still the ulcers were roundish and from a quarter to half an inch in diameter. The right labium was swollen and red, and so was the abdomen for a short distance between the ulcers, but the child seemed lively and well and not in pain until the thigh and groin ulcerated. A sister, æt. 16 years, was attending at the City Road Hospital for consumption, and this child had a bad cough, but there were no physical signs in the chest. On December 1st a small pustule with red areola appeared on the left cheek exactly similar to those in the groin, which were healing. The subsequent history is unknown.

The next one differs in many ways from the preceding vOL. LXX. 
cases and is possibly of tropho-neurotic origin ; it is, however, worthy of record as a local phagedænic ulceration.

CASE 5.-William M-, æt. 9 years, admitted as outpatient at Shadwell on April 30th, 1883. His general health had always been good, and he had had no previous illness up to a week before Christmas, when be was exposed to a chill ; he seemed languid, and then, without pain preceding it, a "blister" the size of a farthing appeared one inch below the middle of the clavicle, which broke and left an open sore, and this spread to a wound three and a half inches by two and three quarters in extent and of irregular shape. Just above this was a superficial oval ulcer about an inch in its long diameter, and below there was a serpentine ulceration, beginning to the right of the middle line and extending irregularly for about an inch and a half to the left of it, but nowhere more than a quarter of an inch broad.

Since the ulceration his head has been in the position of right torticollis and he seems languid but is otherwise tolerably well. The ulcer took nearly six weeks to attain its full size and did not begin to heal for some time after that, but had been well for some weeks when seen by me.

The small patch under the right clavicle was still livid red, and without sensation. The large patch was also deficient in sensibility, a pin-prick being felt as something sticking in, but there was no pain. He was put on iron and cod-liver oil, and gradually recovered, but it was four months before he was restored to health, and the skin of the scar was slowly regaining its natural colour, but there was even then defective sensibility in the cicatrix.

Hilbert ${ }^{1}$ records two cases of spontaneous gangrene of the eyelids in female infants under one year old. A small pustule with yellow scab first formed without apparent cause on the upper lid, rapidly enlarged, the part beneath became gangrenous, and when the slough separated a circular ulcer nearly an inch in diameter was left, which

1 ' Viertelj. f. Derm. u. Syph.,' vol. xi, 1884, p. 117. 
healed rapidly. Both children were healthy and well nourished.

It will occur to everyone that the first four of this set of cases are referable to noma, especially as two of them came on after measles, but granting that this is so it will be noticed that they all began as vesicles or pustules, and from that went on to ulceration and sloughing. So far they therefore corroborate my statement that vesicular eruptions, other than those of varicella and vaccinia, may be the starting-point of gangrenous ulcers.

In a case of Dr. Barlow's which he was kind enough to show me recently, besides the deep punched-out ulcers of the vulva and groin, there were some scabbed spots like dried-up pustules sparsely scattered about the trunk and limbs, and although no history of varicella could be obtained Mr. Hutchinson considered that varicella was at the bottom of it. Even if that were so in Dr. Barlow's case (which I do not believe) it certainly was not in all of mine. On the other hand, there was a strong suspicion of tuberculosis in three of the cases. There are other analogies with cancrum oris, moreover, which may start from quite a superficial ulcerative stomatitis, and this, too, is more frequent in girls than boys, and rarely begins after the sixth year. "Broncho-pneumonia is very common, and pyæmic abscesses have been found in the lungs" (Eustace Smith), and the pneumonia preceded or followed the gangrene. Meigs and Pepper and others have drawn attention to the predisposing influences to it of the tubercular diathesis, while Barthez and Rilliet describe it as beginning with ulceration, aphthæ, oriphlyctenæ of the mucous membrane and only rarely with cedema. I do not of course ignore the fact that vesicles or bullæ are often the first sign of a gangrenous inflammation, but these are of different characters, and I am equally convinced that even when arising from otherwise harmless causes they may be the starting-point of gangrene in tubercular or otherwiso predisposed subjects. 
The conclusions I would draw from the consideration of these cases are :-

1. That infants and children under three years old are liable to multiple gangrenous ulcers.

2. That while the gangrenous lesions most frequently follow those of varicella and vaccinia they may also ensue from other pustular and non-specific lesions under certain conditions.

3. That when once the gangrenous process has started the fresh lesions do not necessarily arise from a pre-existing pustule but may come independently.

4. While the original eruption varies much, the gangrenous lesions are very similar in their course and development, viz. of vesicle, pustule, scab and slough surrounded by a red areola closely resembling, before the slough has formed, a vaccine lesion.

5. That the general conditions which coexist are not only specific fevers, such as varicella, vaccinia, and, for the local forms, measles and scarlatina, but tuberculosis and, perhaps, rickets, which are probably related etiologically to the gangrenous lesions.

6. The one feature that these diseases have in common is the febrile state, but while it is very probable that antecedent fever may be a predisposing cause we are not yet in a position to say that it is essential or that other factors are not required for the production of the gangrene.

Finally, I have only to express my regret that the record of some of the cases is less complete than could be desired, but they were nearly all out-patients, and only seen once or twice, and in the high pressure at which that sort of work has to be done, details which would find a place in a more leisurely record are apt to be omitted.

(For report of the discussion on this paper, see ' Proceedings of the Royal Medical and Chirurgical Society,' New Series, vol. ii, p. 282.) 